\title{
A Success Story of Complicated Antipartum Hemorrage and Placenta Previa in ICU Setting
}

\author{
Ashraf S*, Sebtain A, Iqbal Z, Laiq N, Jawad and Abid \\ Department of Anesthesiology \& Intensive care Lady Reading Hospital, Pakistan
}

*Corresponding author: Sheharyar Ashraf, Department of Anesthesiology \& Intensive care Lady Reading Hospital, Peshawar, Pakistan.

To Cite This Article: Ashraf S, Sebtain A, Iqbal Z, Laiq N, Jawad and Abid, A Success Story of Complicated Antipartum Hemorrage and Placenta Previa in ICU Setting. Am J Biomed Sci \& Res. 2020 - 11(3). AJBSR.MS.ID.001639. DOI: 10.34297/AJBSR.2020.11.001639.

Received: 䟧 November 30, 2020; Published: 眥 December 22, 2020

\begin{abstract}
A 30-year-old woman with 34 weeks pregnancy, with three previous live pregnancies, normotensive and normoglycemic was presented to gene unit with severe per vaginal bleeding, which later on was complicated by multi organ failure. She was shifted to Intensive Care Unit (ICU) with multiple co morbidities including sepsis, hypovolemia, acute renal failure, unilateral pleural effusion, and respiratory insufficiency. She was given 15 sessions of haemodialysis, 15 units of blood transfusions and multiple times wound re-opening for treating wound infection. Inter disciplinary approach by involving nephrology, pulmonology, dermatology, acute medicine, hematology, and vigorous physical rehabilitation stabilized her condition with a good outcome.
\end{abstract}

\section{Introduction}

Antepartum hemorrhage (APH) in Placenta previa (PV) is one of the leading causes of maternal mortality and morbidity in pregnant women around the world [1]. APH is defined as bleeding from the genital tract in the second half of pregnancy. Placenta Previa is a condition in which the placenta is abnormally implanted in the lower uterus, sometimes covering the whole or partial cervical os [2]. It has an overall prevalence in North America of 2.9 per 1000 pregnancies, compared with a global prevalence of 5.2 per 1000 pregnancies [3]. The highest prevalence internationally is in Asian women, in whom the overall prevalence is 12.2 per 1000 pregnancies [4]. The rising incidence of c-sections in the last 50 years is partially a causative factor to the increasing number of cases of placenta previa [5]. Other causes of a placenta previa may include fibroids, large placental area with twins, late fertilization, internal scarring from previous c-sections, smoking and in-vitro fertilization [6]. Apart from APH, many other complications like fetal anemia, distress, shock, infection and renal insufficiency may occur [7]. One of the most important cause of pregnancy induced renal failure is hypo-perfusion that may be triggered by APH which is why, it is important to limit the ongoing damage timely to prevent any irreversible damage to the vital organs [8].

\section{Case Report}

Our case report involved a 30-year-old lady from gyne unit with APH secondary to type 4 placenta previa. On arrival to ICU, she was irritable, hypoxic with GCS of $7 / 15$. As she continued to de saturate (SPO2 50\%), she was started on CPAP but with worsening arterial blood gases (ABGs) i-e; Ph of 6.9, HCO3 of 10mmol, PCO2 of $12 \mathrm{mmHg}$ and $\mathrm{PO} 2$ of $58 \%$ was in severe metabolic acidosis, Hemodynamic instability bp 80/50 and Disseminated Intravascular Coagulation (DIC) were the reason that she was intubation. platelet count was 30,000, haemoglobin Hb of 6, tlc 30000, ALT of 1800, Bilirubin 18 and deranged renal profile she went into multi organ failure (MOF) secondary to sepis (raised BUN and Lactate). On 3rd day of admission to ICU Renal profile was severly deranged i.e urea 410 and creatinine 11 and was Aneuric and labelled as Acute kidney Injury (AKI). Nephrology team was consulted, nephrotoxic drugs were stopped, and she was started on haemodialysis. Almost 15 sessions of dialysis kidney profile start improving with adequate urine output. C section wound was infected and was reopened by involving gynea team. 8th day of ICU admission, she developed acute lung injury due to septic wound. Her coagulation profile shows D Dimers of 2400 and PT 40sec, and INR 2, so fresh frozen 
plasma (FFPs) and platelet transfusion were done. She remained ventilated on volume control mode with fio 2 100\% PEEP 6 Tidal volume 450 , I $\backslash \mathrm{E} 1: 2$. weaning off became so challenging for the ICU team because of ALI. Physical therapist and other mandatory rehabilitation protocols were followed. She started responding to Synchronized mode (SIMV). Broad spectrum antibiotics Colostine and Polymixin were started according to pus culture collected from opened wound which showed Acinetobacter and gram negative bacteroid. wound was closed on her 25th day in ICU after culture and sensitivity of blood, tracheal, urine and wound got negative. GCS gradually improved to $10 / 10$ on 13 day. urine output was in negative balance of $100 \mathrm{ml}$. Topical antibiotics were also added due to valvulitis and patient attendants were educated for hygiene maintenance. Weaning off was started and sessions of bipap were given 2 hours a day. she was given trails of SPONT ventilation and then T-piece trial with $2 \mathrm{~L}$ of oxygen which she initially could not tolerate but later she weaned off completely from mechanical ventilation.

\section{Discussion}

Successful management of AKI secondary to complicated placenta previa requires a multidisciplinary approach with close collaboration among nephrologists, obstetricians, intensivists, and other team members [9]. The identification of the underlying etiology of AKI is notalways straightforward and very much depends on timing and risk factors [10]. Conventional management for APH and placenta previa alone is easy to administer but complicated by several co morbidities made it difficult for the clinicians to prepare the right plan of treatment. One of this patient's primary health concerns was haemodynamic instability. The Nephrology team intervened but that couldn't give satisfactory outcomes. Studies have shown that fluid overload is independently associated with increased morbidity in critically ill [11]. We were trying to manage sepsis and DIC that required fluids and blood transfusions on the other hand this patient had ALI and fluids could have brought hazardous outcomes. So, the fluid resuscitation was strictly followed along with all the biochemical investigations to prevent any harm. Muscle wasting in this patient was very progressive, yet we could not go for protein rich diet because of her AKI therefore we decided to start total parenteral nutrition TPN. Breathing and living on mechanical ventilation develops a different feeling in patients [12]. Most of such feelings and experiences are not pleasant and can lead to psychiatric disorders in these patients [13]. also, patients with longer stay of admission in ICU develop ICU acquired myopathies [14]. Therefore, vigorous physical therapy protocols, mobilization sessions and counselling helped her recover from ICU acquired myopathies and psychosis. Respiratory muscle weakness resulting into ventilator dependency was the major challenge to address. She was given tri-flo exercises, strengthening, weaning off trials to which she very slowly started to respond. Her overall prognosis was not that speedy, however, the interdisciplinary approach and timely management of the co morbidities were the key reason for her successful recovery.

\section{Conclusion}

Placenta previa with acute tubular necrosis is the most notorious morbidity with high fatality rates. The rising incidence of c section for placenta previa is the most common cause of acute kidney injury. Initial timely treatment can minimize the comorbidities and complications. The primary cause of a disease getting complicated is the lack of multi-disciplinary approach towards the better prognosis and this lack can result as a great risk factor in increasing case fatality.

\section{References}

1. Lala ABH, Rutherford JM (2002) Massive or recurrent antepartum haemorrhage. Current Obstet Gynaecol 12(4): 226-230.

2. Marshall NE, Fu R, Guise JM (2011) Impact of multiple cesarean deliveries on maternal morbidity: a systematic review. Am J Obstet Gynecol 205(3): 262.

3. Milosević J, Lilić V, Tasić M, Radović Janosević D, Stefanović M, et al (2009) [Placental complications after a previous cesarean section] Med Pregl 62(5-6): 212-216.

4. Faiz AS, Ananth CV (2003) Etiology and risk factors for placenta previa: an overview and meta-analysis of observational studies. J Matern Fetal Neonatal Med 13(3): 175-190.

5. Betrán AP, Merialdi M, Lauer JA, Bing-Shun W, Thomas J, et al (2007) Rates of caesarean section: analysis of global, regional and national estimates. Paediatr Perinat Epidemiol 21(2): 98-113.

6. Stavrou EP, Ford JB, Shand AW, Morris JM, Roberts CL (2011) Epidemiology and trends for Caesarean section births in New South Wales, Australia: a population-based study. BMC Pregnancy Childbirth 11: 8 .

7. Blackwell SC (2011) Timing of delivery for women with stable placenta previa. Semin Perinatol. 35(5): 249-251.

8. Räisänen S, Kancherla V, Kramer MR, Gissler M, Heinonen S (2014) Placenta previa and the risk of delivering a small-for-gestational-age newborn. Obstet Gynecol 124(2 Pt 1): 285-291.

9. Kuklina EV, Ayala C, Callaghan WM (2009) Hypertensive disorders and severe obstetric morbidity in the United States. Obstet Gynecol 113(6): 1299-1306.

10. Lam C. Wong SF, Chow KM, Ho LC (2000) Women with placenta praevia and antepartum haemorrhage have a worse outcome than those who do not bleed before delivery. J Obstet Gynaecol 20(1): 27-31.

11. Gorodeski I. Neri A, Bahary CM (1985) Placenta previa-the identification of low-and high-risk subgroups. Eur J Obstet Gynecol Reprod Biol 20(3): 133-143.

12. Sekiguchi A, Nakai A, Kawabata I, Hayashi M, Takeshita T (2013) Type and location of placenta previa affect preterm delivery risk related to antepartum hemorrhage. Int J Med Sci 10(12): 1683-1688.

13. Wortman A. Wortman AC, Twickler DM, McIntire DD, Dashe JS (2016) Bleeding complications in pregnancies with low-lying placenta. J Matern Fetal Neonatal Med 29(9): 1367-1371.

14. Curti A. Potti S, Di Donato N, Simonazzi G, Rizzo N, et al (2013) Cervical length and risk of antepartum hemorrhage in presence of low-lying placenta. J Matern Fetal Neonatal Med 26(6): 563-565. 